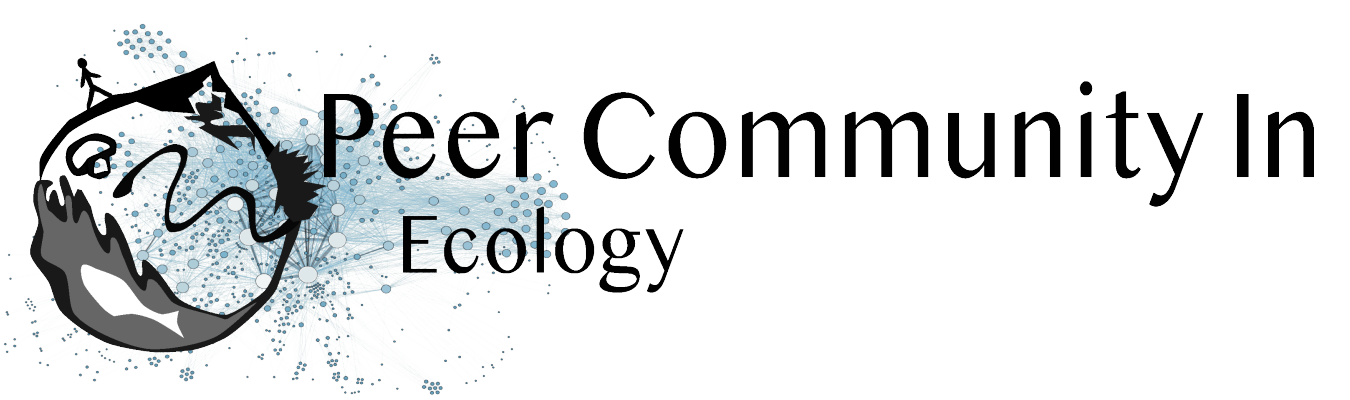

Open Access

\title{
RECOMMENDATION Some thoughts on computational ecology from people who I'm sure use different passwords for each of their accounts
}

Cite as: Staniczenko PP. Some

thoughts on computational ecology

from people who I'm sure use different passwords for each of their accounts. Peer Community In Ecology, 100002 (2018). DOI:

10.24072/pci.ecology.100002

Published: 1st June 2018

\section{Based on reviews by:}

Matthieu Barbier

anonymous

Correspondence:

phillip.staniczenko@gmail.com

\author{
Phillip P.A. Staniczenko
}

National Socio-Environmental Synthesis Center, University of Maryland, College Park - Annapolis, United States of America

\footnotetext{
A recommendation of

Poisot T, Labrie R, Larson E, and Rahlin A. Data-based, synthesis-driven: setting the agenda for computational ecology. bioRxiv 150128, ver 4 peer-reviewed and recommended by PCI Ecology (2018). DOI: $10.1101 / 150128$
}

Are you an ecologist who uses a computer or know someone that does?

Even if your research doesn't rely heavily on advanced computational techniques, it likely hasn't escaped your attention that computers are increasingly being used to analyse field data and make predictions about the consequences of environmental change. So before artificial intelligence and robots take over from scientists, now is great time to read about how experts think computers could make your life easier and lead to innovations in ecological research. In "Data-based, synthesis-driven: setting the agenda for computational ecology", Poisot and colleagues [1] provide a brief history of computational ecology and offer their thoughts on how computational thinking can help to bridge different types of ecological knowledge.

In this wide-ranging article, the authors share practical strategies for realising three main goals: (i) tighter integration of data and models to make predictions that motivate action by practitioners and policy-makers; (ii) closer interaction between data-collectors and data-users; and (iii) enthusiasm and aptitude for computational techniques in future generations of ecologists.

The key, Poisot and colleagues argue, is for ecologists to "engage in meaningful dialogue across disciplines, and recognize the currencies of their collaborations."

Yes, this is easier said than done. However, the journey is much easier with a guide and when everyone involved serves to benefit not only from the eventual outcome, but also the process. 


\section{References}

[1] Poisot T, Labrie R, Larson E, and Rahlin A. Data-based, synthesis-driven: setting the agenda for computational ecology. bioRxiv 150128, ver 4 peer-reviewed and recommended by PCI Ecology (2018). DOI: 10.1101/150128.

\section{Appendix}

Reviews by Matthieu Barbier and anonymous, DOI: 10.24072/pci.ecology.100002 